\title{
Les prieurés de Château-Gontier et l'établissement d'une seigneurie châtelaine dans le comté d'Anjou (fin du Xe siècle-fin du XIe siècle)
}

\section{Sébastien Legros}

\section{(2) OpenEdition \\ Journals}

Édition électronique

URL : http://journals.openedition.org/abpo/772

DOI : $10.4000 /$ abpo. 772

ISBN : 978-2-7535-1503-1

ISSN : 2108-6443

\section{Éditeur}

Presses universitaires de Rennes

\section{Édition imprimée}

Date de publication : 30 octobre 2006

Pagination : $33-59$

ISBN : 978-2-7535-0405-9

ISSN : 0399-0826

\section{Référence électronique}

Sébastien Legros, «Les prieurés de Château-Gontier et l'établissement d'une seigneurie châtelaine dans le comté d'Anjou (fin du Xe siècle-fin du Xle siècle) ", Annales de Bretagne et des Pays de l'Ouest [En ligne], 113-3 | 2006, mis en ligne le 30 octobre 2008, consulté le 20 avril 2019. URL : http:// journals.openedition.org/abpo/772 ; DOI : 10.4000/abpo.772 


\title{
Les prieurés de Château-Gontier et l'établissement d'une seigneurie châtelaine dans le comté d'Anjou (fin du $\mathrm{X}^{\mathrm{e}}$ siècle-fin du $\mathrm{XI}^{\mathrm{e}}$ siècle)
}

\author{
Sébastien LEGROS \\ Doctorant en Histoire, Université Rennes 2 Haute-Bretagne \\ CRHISCO, CERHIO, FRE CNRS 3004
}

\begin{abstract}
"C'est en l'an de l'incarnation du Seigneur 1007, indiction V, que naquit Geoffroy Martel et que son père Foulque, le très noble comte des Angevins, fils du très fort comte Geoffroy Grisegonelle, établit un château sur la Mayenne, dans la cour de Bazouges. Plusieurs années auparavant il avait cédé cette cour à l'abbé Renaud et aux moines de Saint-Aubin, en échange d'une autre, Houdainville en Beauvaisis, et il la leur avait laissée en possession perpétuelle, avec ses dépendances. Il établit donc ce château et le fortifia autant qu'il put, et il l'appela Château-Gontier, du nom d'un de ses villici ${ }^{1}$... "
\end{abstract}

Ce court extrait de la charte première du Cartulaire de l'abbaye SaintAubin d'Angers, plante en quelques mots le contexte dans lequel s'établit le prieuré Saint-Jean-Baptiste de Château-Gontier. Les moines qui rédigent cette charte en faveur de cet établissement cherchent ici à rapporter son origine à une époque bien précise et à la lier à deux personnages essentiels : le dépositaire du château comtal et le comte des Angevins. Entre ces différents protagonistes (les moines, le comte et celui qui allait devenir le seigneur de Château-Gontier), s'établit donc de façon précoce un rapport de rivalité, qui allait largement déterminer le devenir du prieuré castrogontérien.

Il serait intéressant d'évaluer l'originalité de ce rapport, compte tenu des différents modèles d'implantation prieurale constatés par ailleurs,

1. Bertrand de Broussillon, Arthur, Cartulaire de l'abbaye de Saint-Aubin d'Angers, Angers, 1903, notice 1 : " anno ab incarnatione Dei 1007, indict. V, Gaufridus Martellus natus est, et pater ejus Fulco, nobilissimus comes Andegavorum, filius Gaufredi fortissimi comitis qui cognomatus est Grisigonella, firmavit castellum super Meduanam fluvium. In curte quae vocatur Basilica, quam idem ante plurimos annos proquadam curte quae nuncupatur Undanis-Villa in pago Belvacensi sita, Rainaldo abbati et monachis Sancti Albini commutaverat, eisque solidam et quietem eum omnibus ad ipsum pertinentibus in perpetuum possidendam tradiderat " (désormais abrégé C Saint-Aubin, 1). 
autour de Laval ou de Mayenne par exemple. Le cas mayennais constitue un schéma a priori relativement simple, dans lequel le châtelain local, profitant d'une inféodation en bonne et due forme, consolide sa domination par la dotation d'un prieuré dépendant de l'abbaye de Marmoutier : cette fondation intervient en 1059 et donne l'occasion à Geoffroy de Mayenne de recevoir le qualificatif de dominus sous la plume des moines tourangeaux ${ }^{2}$. Le schéma lavallois semble plus complexe : ici le seigneur, installé manu militari vers 1030, cherche à légitimer sa position en gratifiant, là encore, l'abbaye de Marmoutier, à la fois par choix politique mais aussi par choix religieux; en fondant le prieuré Saint-Martin à Laval, vers 1060, Guy I ${ }^{\text {er }}$, en mal de légitimité, obtient ainsi des moines tourangeaux qu'ils cautionnent sa nobilitas et qu'ils soulignent sa participation à leur élan réformateur en recevant son fils Jean en leur sein ${ }^{3}$.

Le schéma castrogontérien présente quant à lui d'immédiates spécificités. Il s'appuie en premier lieu sur une antériorité chronologique évidente. C'est en effet en 974 que la comtesse Adèle de Vermandois, première femme de Geoffroy Grisegonelle, a donné à Saint-Aubin ses biens héréditaires, parmi lesquels « une cour, qui est appelée Houdainville, située dans le pagus de Beauvais ${ }^{4}$ "; cette cour a ensuite été échangée avec le comte d'Anjou, entre 988 et $1001^{5}$, contre la cour de Bazouges, qui est reçue par les moines " libre de toute coutume et exaction " (comme celle d'Houdainville). L'arrivée des religieux précède donc l'érection du château et sa remise à un fidèle de Foulque Nerra, un miles nommé Gontier, vraisemblablement dans la première décennie du XI ${ }^{\mathrm{e}}$ siècle. C'est en tout cas ce que suggère le texte cité en préambule, extrait d'une charte qui, pour " trafiquée " qu'elle ait été dans sa corroboration finale, n'en demeure pas moins vraisemblablement exacte dans le récit qu'elle propose ${ }^{6}$. Il en résulte que la fondation

2. LAURAIn, Ernest, Cartulaire manceau de Marmoutier, t. 2, Laval, 1945, p. 1-2, n 1 (désormais abrégé CMM, Mayenne, 1) : Geoffroy de Mayenne et Hamelin Lavaré, un de ses milites, donnent une terre à Marmoutier, dans le vieux bourg de Mayenne, ainsi que l'église Saint-Martin. Geoffroy est qualifié de : "castri prefati possessorem ac dominum ".

3. CMM, Laval, 1, 2 et 3; nous nous permettons de renvoyer à LEGROS, Sébastien, "Le prieuré Saint-Martin de Laval et la consolidation de la seigneurie lavalloise au $\mathrm{XI}^{\mathrm{e}}$ siècle ", La Mayenne. Archéologie. Histoire (désormais abrégé MAH), 26, 2003, p. 12-43.

4. C Saint-Aubin, 3 : "dono igitur illi curtem a parentibus traditam, sitam in pago Belvacinse, que vocatur Hundanis Villa...".

5. Vraisemblablement du temps de l'abbé Renaud, qui est mort en septembre 1001. Voir C Saint-Aubin, 677, qui relate cet échange entre les moines et le comte Foulque Nerra : " monachi darent comiti curtem quandam in pago Belvacensi consistentem, Undanis villa vocatum, ab omni consuetudine et exactione liberrimam; et comes daret monachis surtem, Basilicas nominatam, in pago Andecavino sitam, similiter liberam et quietam ". Cité en préambule d'une notice des années 1056-1060, cet acte serait antérieur à l'année 1001, selon la datation proposée par GuILLOT, Olivier, Le comte d'Anjou et son entourage au X⿸厃 siècle, Paris, 1972, t. 2, catalogue d'actes, p. 29.

6. BARTHELEMY, Dominique, La mutation féodale a-t-elle eu lieu? Servage et chevalerie dans la France des $X^{e}$ et $X I^{e}$ siècles, Paris, 1996, p. 55, voit en cette charte une rédaction préparatoire à une notice jamais officialisée, mais réutilisée ultérieurement (et frauduleusement) par les moines au prix d'une manipulation du protocole final. Reprenant ces 
du prieuré Saint-Jean-Baptiste ne procède nullement d'une sollicitation seigneuriale ou de la donation du temporel effectuée par un seigneur pré-existant. C'est même plutôt l'inverse, puisque c'est dans la cour des moines que Foulque Nerra construisit son château, au point que "l'aumône faite à Saint-Aubin... en pâtissait beaucoup ${ }^{7}$ ".

Dans ce cadre, notre étude cherchera à relever en quoi cette situation originale détermine les rapports entre le châtelain castrogontérien et les moines de Saint-Aubin. Par suite, il s'agira de voir en quoi cette situation originelle se répercute sur les autres fondations de prieurés autour de Château-Gontier. Les moines de Saint-Aubin furent en effet assez rapidement rejoints par des confrères des abbayes de la Trinité de Vendôme et de Saint-Nicolas, qui s'installèrent les uns à Ménil, les autres à Azé et au Genéteil. Quelle fut l'attitude du comte d'Anjou et du châtelain castrogontérien à l'égard de ces différents prieurés? Comment ces différents établissements, se sont-ils inscrits dans le réseau féodal en cours de constitution? En quoi ces prieurés participèrent-ils à l'émergence de la seigneurie châtelaine de Château-Gontier? Ce sont là les quelques-unes des questions qui nous préoccuperont.

\section{Le cadre de l'implantation monastique}

Avant toute chose, il est utile de présenter brièvement l'environnement dans lequel prirent place nos différents moines. La fondation d'un établissement prieural dépend en effet organiquement du milieu géographique et humain dans lequel cet établissement s'insère. Un panorama rapide de l'environnement castrogontérien aux alentours de l'an mil montre l'ampleur des occupations antérieures et permet d'expliquer l'intérêt du site de Bazouges pour les moines de Saint-Aubin ${ }^{8}$.

À cet égard, les témoignages de l'implantation mérovingienne dans la région castrogontérienne sont relativement importants. La première indication se rencontre à Azé, entre 529 et 550 : saint Aubin visite alors le "monastère d'Azé ", où il rend la vue à un aveugle; c'est l'unique mention

conclusions, PeChA, M., "La notice ${ }^{\circ} 1$ du Cartulaire de Saint-Aubin d'Angers, chronique de la naissance de Château-Gontier ", $M A H, 24,2001$, p. 17 et 22, semble situer cette charte à la toute fin du XI ${ }^{\mathrm{e}}$ siècle, après l'émergence de la seigneurie châtelaine castrogontérienne dans les années 1090; son étude met bien en évidence la falsification du protocole final, sans pouvoir précisément situer l'époque de cette manipulation. En dernier lieu l'analyse d'Olivier Guillot semble la plus probante : se fondant sur une étude du foliotage des neuf premières chartes du Cartulaire de Saint-Aubin d'Angers, il estime que cette charte ne peut être antérieure à l'année 1139 (Guillot O., Le comte d'Anjou..., op. cit., t. 1, appendice 1, p. 440, n. 22).

7. C Saint-Aubin, 1 : «quia elemosynae... Sancto Albino contulerat, damnum maximum intulisse videbatur...".

8. L'installation d'un de ses milites par le comte d'Anjou à Château-Gontier obéit certainement aux mêmes considérations géographiques et humaines : voir sur ce point Pecha, M., "La notice ${ }^{\circ} 1$... ", art. cit., p. 1-32 et spécialement p. 12 (il évoque, pour l'essentiel, le cadre hydrologique et la navigabilité de la Mayenne). 
de ce monastère dont on ne sait quand il disparut ${ }^{9}$. Il atteste que dès le $\mathrm{VI}^{\mathrm{e}}$ siècle une communauté vivait à Azé, où aboutissait d'ailleurs une voie romaine venant d'Entrammes, via Villiers-Charlemagne. Cette implantation n'était localement pas la seule. Le recensement des cimetières mérovingiens en Mayenne récemment réalisé par Rozenn Colleter met en évidence une dizaine de sites locaux, parmi lesquels se distinguent ceux de Loigné et de Ménil ${ }^{10}$; une fouille récente, à Ménil, confirme d'ailleurs, au-delà des attentes, l'importance de ce site en bordure de la Mayenne : on peut penser qu'il constituait un point d'aboutissement pour les sarcophages en calcaire coquillier venus du bassin de la Loire. La dispersion des autres emplacements montrerait, toujours selon Rozenn Colleter, la dispersion des habitats mérovingiens, laquelle serait essentiellement déterminée par la Mayenne et les cours d'eaux secondaires ${ }^{11}$. Ces trouvailles ont le mérite de permettre une cartographie élémentaire de cette occupation, à laquelle on peut utilement associer nos connaissances du réseau des chemins anciens ${ }^{12}$, et que l'on complétera, pour l'époque suivante, par plusieurs indications bien datées. Celles-ci permettent d'éclairer les modalités de l'implantation humaine dans le milieu (voir fig. 1).

Un bref état des lieux montre une occupation différenciée de part et d'autre de la Mayenne. À l'est de la rivière, une seule mention indiquerait la présence d'une propriété de l'évêque du Mans Mérole, à VilliersCharlemagne, en 783, laquelle serait confirmée à l'église du Mans en $832^{13}$; l'attribution reste incertaine, même si divers indices plaident en faveur d'un habitat local ancien ${ }^{14}$. À l'ouest de la rivière, la situation est tout autre avec quatre témoignages de mise en valeur carolingienne. Trois villae

9. Acta sanctorum, p. 54-63.

10. COLLETER, Rozenn, Les cimetières mérovingiens en Mayenne, Laval, 2003 (voir le catalogue des sites, p. 53-189).

11. Ibidem, p. 66; le site de la Françoinière, à Loigné, constitue également un site majeur. Nous renvoyons également à NAVEAU, Jacques, Carte archéologique de la Gaule. La Mayenne, Paris, 1992, ainsi qu'à notre carte pour les localisations des différents sites répertoriés, qui sont localisés comme suit : Daon, jardin de l'Hôpital, 2 sarcophages; Grezen-Bouère : la Daguinière, plusieurs sarcophages (site douteux); Houssay, église, plusieurs sarcophages; Loigné, église, plusieurs sarcophages; Loigné, la Françoisière/l'Erable, plusieurs sarcophages avec du matériel des IVe-ve siècles; Ménil, bourg, une vingtaine de sarcophages; Quelaines, la Goguerie, 8 sarcophages; Saint-Sulpice, Neuville, plusieurs sarcophages, Saint-Sulpice, la Querlière, nombreux sarcophages; Villiers-Charlemagne, église, 2 sarcophages. En l'absence de mobilier, la datation de ces sarcophages est le plus souvent au mieux aléatoire, au pire impossible.

12. À cet égard les remarques de l'abbé VoIsIN, "Les Basouges de Château-Gontier ", dans Bulletin monumental, 5,1869 , p. 192-194 ont été consultées avec d'infinies précautions, notamment parce que l'auteur ne date aucune de ses informations.

13. Voir, ChARLES, R. et Froger, Louis, Gesta domini Aldrici cenomannicae urbis episcopi a discipulis suis, Le Mans, 1889, p. 183, qui évoque un lieu nommé "Villare ", sans autre précision (cf. ANGOT, Alphonse, Dictionnaire historique, topographique et bibliographique de la Mayenne, Laval, 1900-1909, 4 vol., t. 3, p. 910).

14. Une voie romaine passe ainsi près de l'église où deux sarcophages de calcaire coquillier ont été découverts tandis que d'autres ont été réutilisés lors de la construction de l'édifice. 
Figure 1 - Bazouges dans son environnement pré-féodal

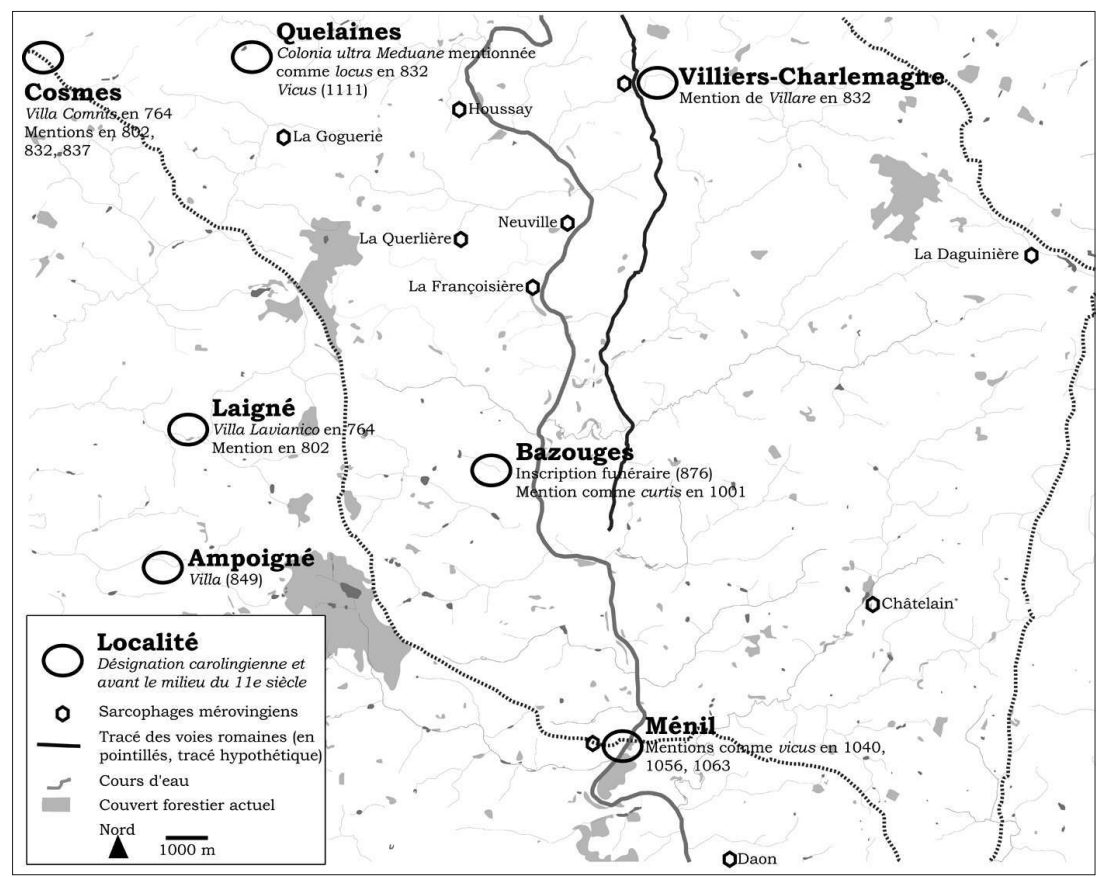

apparaissent ainsi à Cosmes, Laigné et Ampoigné, dans la première moitié du IX ${ }^{\mathrm{e}}$ siècle, au moment où elles entrent dans les patrimoines des Églises du Mans et d'Angers; plus au nord, Quelaines apparaît comme un simple locus, mentionné dans le testament de l'évêque du Mans Aldric, en $847^{15}$.

15. Sur Cosmes, voir dom MARTÈnE, Amplissima collectio, Paris, 1724, p. 28-30 : en 764 un certain Egidius, devenant moine de l'abbaye de Prüm lui avait donné diverses propriétés en territoire angevin, dont la "villa Comnis "; l'église du Mans dispose également de propriétés à Cosmes, qui lui sont confirmées par Charlemagne et Louis le Pieux en 802 et 832 (CAuvin, Thomas, Géographie ancienne du diocèse du Mans, Paris, 1845, p. 55 et 273; Cosmes est peut-être alors désignée comme une villula) tandis que saint Aldric entretient des troupeaux « in Comnis » en 837 (ChARlES, R. et Froger, Louis, Gesta domini Aldrici, op. cit., p. 103). À Laigné, Egidius dispose là aussi de sa "villa Lavianico " en faveur de Prüm, tandis que Charlemagne confirme des biens en faveur de l'église du Mans en 802 (éd. MARTĖNE, Amplissima collectio, op. cit., p. 28-30 et 60-61). À Ampoigné, Charles le Chauve donne une villa à Saint-Maurice d'Angers en 849 (ANGOT, Alphonse, Dictionnaire historique..., op. cit., t. 1, p. 37). Le cas de Quelaines est plus hypothétique : dans son testament l'évêque Aldric mentionne "diversis mansionilibus et locis " parmi lesquels "Colonia ultra Meduana " (Charles, R. et Froger, Louis, Gesta domini Aldrici, op. cit., p. 105); l'identification avec Quelaines est possible : dom Piolin, Histoire de l'église du Mans, Le Mans, 1851-1871, t. 3, p. 580 la fait, qualifiant Quelaines de bourg public (voir aussi Pıснот, Daniel, Le Bas-Maine du Xe au XIII siècle : étude d'une société, Laval, 1995, p. 53, n. 93). 
On remarquera que ces diverses mentions s'étalent, pour l'essentiel, à l'ouest de la voie romaine Avranches-Angers, dont nous restituons, sur la carte, un tracé hypothétique aimablement communiqué par Jacques Naveau. Cette voie conduit vraisemblablement vers le "Gué de Mesnil ", où elle franchit la Mayenne, et poursuit son chemin vers Sœurdres. Le franchissement de Ménil est intéressant : le passé mérovingien de cette localité et sa qualification comme vicus vers 1040, au moment de l'arrivée vraisemblable des moines de la Trinité de Vendôme ${ }^{16}$, oblige à s'interroger sur l'état carolingien du site. Les fouilles à venir de la nécropole récemment mise au jour sont à ce titre très attendues. Mais plus que le réseau de chemins, c'est sans doute le milieu naturel qui explique la répartition des implantations. Le caractère forestier de la région est en effet relativement important, sans doute même omniprésent autour des actuelles forêts de Rouillières et de Valles. On sait quelles précautions doivent être prises dans la reconstitution du couvert haut-médiéval ${ }^{17}$, mais un relevé des toponymes forestiers laisse entrevoir une certaine cohérence indiquant vraisemblablement sa prégnance ${ }^{18}$. Au nord de notre zone, le couvert forestier semble particulièrement important à Cosmes, Peuton et Marigné, comme en témoigne d'ailleurs ultérieurement le Cartulaire de la Roë ${ }^{19}$. Ce couvert s'étendait-il de façon continue vers le sud, en direction de Ménil, suivant le léger affleurement (entre 90 et 100 mètres) qui abrite les forêts de Rouillières et de Valles, au pied duquel se déroule la voie romaine évoquée plus haut? C'est l'impression que donnerait la carte, sans que cela soit une certitude. Si nous suivons cette hypothèse la curtis de Bazouges s'insérerait entre la Mayenne et cette écharpe forestière longée par une voie ancienne, au-delà de laquelle les Églises du Mans et d'Angers disposeraient de plusieurs domaines.

Le site de Bazouges constituait lui-même, comme le soulignait Louis Halphen, une ancienne possession du comte d'Anjou, qui relevait directe-

16. MÉTAIS, Charles, Cartulaire de l'abbaye cardinale de la Trinité de Vendôme, Paris, 18931895, n 36 et 38 (désormais abrégé C TV, 36 et 38; voir aussi C TV, 107 et 164). Pour une discussion du terme vicus, voir Ріснот, Daniel, Le village éclaté. Habitat et société dans les campagnes de l'Ouest au Moyen âge, Rennes, 2002, p. 47-48.

17. Voir à cet égard, pour le Bas-Maine, les résultats de la récente thèse de palynologie de BARBIER, D., Histoire de la végétation du Nord-mayennais de la fin du Weichsélien à l'aube du XXI siècle, Nantes, 2002.

18. Nous avons procédé à un relevé cartographique des toponymes intégrant les termes suivants : forêt, bois, plessis, futaie, taillis, haie, touche et ceux évoquant une essence : chêne, chênaie, hêtre, cormier, frêne, aulne.

19. Pour Cosmes, mention en 1149 du Bois de la Métairie (C Roë, $\mathrm{f}^{\circ} 63 \mathrm{r}^{\circ}$ ), puis mention entre 1150 et 1170 du Grand-Bois, près de la Bélinière (C Roë, $f^{\circ} 72 r^{\circ}$ ) et de la haie ou de la barre de Thorigné (C Roë, $\mathrm{f}^{\circ} 64 \mathrm{r}^{\circ}-\mathrm{v}^{\circ}$ et $\mathrm{f}^{\circ} 72 \mathrm{r}^{\circ}-73^{\circ}$ ). Pour Peuton, qui est d'ailleurs désigné sous les termes de Bois-Peuton (C Roë, $\mathrm{f}^{\circ} 41 \mathrm{v}^{\circ}, 44 \mathrm{r}^{\circ}, 49 \mathrm{r}^{\circ}, 58 \mathrm{r}^{\circ}, 75 \mathrm{r}^{\circ}$ $76 \mathrm{r}^{\circ}$ entre 1128 et 1170 ), mention entre 1150 et 1170 du bois de la Rapinière (C Roë, $\left.\mathrm{f}^{\circ} 62 \mathrm{v}^{\circ}\right)$ et du Bois d'Aulnay $\left(\mathrm{C}\right.$ Roë, $\left.\mathrm{f}^{\circ} 54 \mathrm{r}^{\circ}-\mathrm{v}^{\circ}\right)$. Ajoutons que le Cartulaire de Saint-Aubin mentionne le Bois de Fay en 1181 (C Saint-Aubin, 684) à quoi s'ajoute une mention du bois de Cornouailles en 1310 (Arch. dép. de Mayenne, H 147, f ${ }^{\circ}$ 71) : ces deux bois bordent la forêt de Valles. 
ment de sa domination jusqu'à la fin du $\mathrm{x}^{\mathrm{e}}$ siècle $^{20}$. Cette curtis Basilica ne nous est malheureusement pas décrite, au contraire de celle d'Houdainville contre laquelle elle est échangée. L'ampleur de cette dernière laisse toutefois peut-être indirectement entrevoir celle de Bazouges ${ }^{21}$. À cet égard, l'échange impliquait vraisemblablement la remise d'une église, qui serait la plus ancienne attestée localement, et dont le nom détermine d'ailleurs celui de Bazouges (basilica). Sa présence est assurée par le préambule de l'acte n 677 du Cartulaire de l'abbaye Saint-Aubin, dont Olivier Guillot a démontré l'antériorité à l'année $1001^{22}$. Cette datation pourrait permettre d'atténuer les hésitations de l'abbé Angot à l'égard de cette église, dont il refusait d'assurer l'antériorité par rapport à l'établissement des moines ${ }^{23}$. L'association étroite entre curtis et ecclesia plaide pourtant en ce sens ${ }^{24}$. Une inscription funéraire en l'honneur d'un certain Giswhal, décédé en 876, découverte en 1835 dans le sol d'un bas-côté de l'église de Bazouges, pourrait venir étayer cette hypothèse : bien qu'on ignore l'emplacement initial de cette inscription, sa localisation dans l'église de Bazouges suggère la présence, sinon d'un lieu de culte, du moins d'un cimetière et d'une communauté humaine dans le courant du $\mathrm{IX}^{\mathrm{e}}$ siècle ${ }^{25}$. L'identification archéologique récente d'une exploitation carolingienne au lieu de Vauvert, près de l'actuelle Bazouges, vient d'ailleurs appuyer cette hypothèse : ses fossés ont permis de la dater, sans qu'on puisse malheureusement aller beaucoup plus loin dans l'analyse, dans la mesure où la partie la plus intéressante du site n'a pu être fouillée ${ }^{26}$... À tout le moins, sa présence confirme-t-elle une occupation carolingienne, dont la curtis du $\mathrm{IX}^{\mathrm{e}}$ siècle est vraisemblablement l'héritière.

Voilà donc le cadre dans lequel les moines de Saint-Aubin viennent s'installer aux alentours de l'an mil : l'occupation locale est visiblement ancienne et profite notamment aux Églises du Mans et d'Angers, présentes à l'ouest d'un massif boisé sans doute important. Bordé à l'est par une antique voie menant vers le gué de Ménil, ce massif entoure peut-être Bazouges d'une écharpe forestière, au point que l'on peut sans doute voir une part de vrai dans la remarque du comte Foulque le Réchin qui, évoquant la région, parlait d' " une terre qui était restée déserte et couverte de bois à cause des courses des païens ${ }^{27}$ ".

20. HaLPHEN, Louis, Le comté d'Anjou au XI siècle, Paris, 1906, p. 16, n. 2.

21. C Saint-Aubin, 3 : la comtesse donne une description classique "curtis... qui est appelé Houdainville, avec des terres cultes et incultes, des villulae, des esclaves des deux sexes, des prés, bois, étangs et eaux courantes, des moulins et avec deux églises ".

22. C Saint-Aubin, 677; pour la datation, voir ci-dessus n. 1.

23. AnGoT, Alphonse, Dictionnaire historique..., op. cit., t. 1, 1909, p. 191. Nous précisons que l'abbé Angot situait l'érection de cette église au titre paroissial au milieu du Xle siècle au plus tôt, et notait, p. 188, que la première mention de la "parochia de Bazogeyo " date de 1283.

24. BARTHELEMY, Dominique, La société dans le comté de Vendôme de l'an mil au XIVe siècle, Paris, 1990, p. 454 et 467.

25. AnGot, Alphonse, Épigraphie de la Mayenne, Laval, 1907, p. 68 et planche 103.

26. Cette partie est aujourd'hui dissimulée par un giratoire.

27. Cité par Godard FAultrier, L'Anjou et ses monuments, t. 1, p. 392. Il évoque ici sans doute les incursions normandes et bretonnes. 
Dans ce cadre, la cour de Bazouges présente certainement l'intérêt d'abriter un noyau de peuplement stable autour d'une église où les moines pourront remplir leur service religieux. Ils s'y installent dans un contexte qui leur est par ailleurs favorable, à la suite de l'achèvement de la réforme de leur abbaye entreprise de 929 à 966 : sur le plan temporel les terres de l'abbaye doivent en effet, à présent, être séparées du patrimoine comtal, ce qui permet aux moines angevins une administration plus sereine et plus directe $^{28}$. S'il faut évidemment juger cette mesure à son usage, toujours estil que les donations sont alors importantes et que le patrimoine s'accroît nettement : l'installation à Bazouges, un peu avant l'an mil, s'inscrit dans ce développement ${ }^{29}$.

\section{Les moines face au château comtal}

Il reste à présenter la forme prise par l'installation des moines à Bazouges. On ne saurait évidemment parler de "prieuré ": le terme ne se rencontre d'ailleurs dans la documentation relative à Saint-Jean-Baptiste qu'en 1283, ce qui est assez tardif. En la matière, nous ne pouvons suivre l'évolution terminologique qu'à travers un nombre limité de documents ${ }^{30}$. La désignation de l'établissement comme curtis désigne vraisemblablement la possession matérielle de l'église de Bazouges et ses dépendances ${ }^{31}$, lesquelles déterminent une unité seigneuriale qui sera finalement désignée comme telle en 1109. Le seigneur de Château-Gontier reconnaît alors " que la cour seigneuriale de Bazouges [curtem dominicam], que les moines de Saint-Aubin possèdent près de Château-Gontier " est libre de toute coutume. Si cet acte reconnaît certains droit aux moines, il entérine aussi la présence, à leur côté, d'un voisin initialement inexistant : le seigneur de Château-Gontier. L'émergence de ce voisinage inattendu nous ramène à une intervention initiale du comte d'Anjou, dans le contexte passablement embrouillé qui suit la remise de la cour de Bazouges aux religieux. Regardons de plus près cette situation, au demeurant fort mal connue.

\footnotetext{
28. Voir spécialement les pages éclairantes de GuILLot, Olivier, Le comte d'Anjou..., op. cit., p. 137-149.

29. HALPHEN, Louis, Le comté d'Anjou au XI siècle, Paris, 1906, p. 89-90 : l'abbaye acquiert les domaines des Alleux, du Lion d'Angers, de Craon, de Vaux, de Saillé, de la Pèlerine et de Bazouges aux alentours de l'an mil.

30. Pour cette question terminologique, voir BAUTIER, Anne-Marie, "De "prepositus" à "prior", de "cella" à "prioratus" : évolution linguistique et genèse d'une institution (jusqu'à 1200) ", dans Prieurs et prieurés dans l'Occident médiéval, Paris, 1987, p. 14-15. Notons que l'établissement de Bazouges ne nous laisse que 8 actes pour l'ensemble du Xie siècle, contre 23 pour un prieuré comparable comme celui de Laval; cet établissement est qualifié de "prieuré " assez rapidement, entre 1155 et 1164 (Cartulaire manceau de Marmoutier, Laval, 21).

31. Cf. C. Saint-Aubin : «[...] curte quae vocatur Basilica, quam idem ante plurimos annos proquadam curte quae nuncupatur Undanis-Villa..., monachis Sancti Albini commutaverat, eisque solidam et quietem eum omnibus ad ipsum pertinentibus ".
} 
Les premiers temps de l'établissement de Bazouges ne sont évoqués que par trois documents couvrant grosso modo une soixantaine d'années. Le premier est constitué par la charte $n^{\circ} 677$ du Cartulaire de Saint-Aubin d'Angers, dont la première partie, qui relate l'échange initial apportant la cour de Bazouges aux moines, est antérieure à l'année 1001. Le reste du texte date vraisemblablement des années 1056-1060 : il décrit l'attitude de Foulque Nerra et de Geoffroy Martel à l'égard de la terre de Bazouges et montre comment l'un et l'autre ont spolié le dominium des moines. Ils le reconnaissent finalement (en partie seulement) à travers l'approbation d'une restitution intervenue en 1056-1060. L'acte n ${ }^{\circ} 676$ du cartulaire s'associe à cette dernière restitution : il relate la donation réalisée par un certain Otbert, en 1056-1060, à travers laquelle le comte reconnaît, là encore, le dominium des moines. Le troisième acte relate quant à lui, l'installation d'un château puis d'une tour, par le comte, sur les terres de la curtis des moines : il s'agit de la charte $n^{\circ} 1$ du cartulaire ${ }^{32}$.

L'étude de ces trois actes permet d'identifier sept moments chronologiques distincts de la vie de l'établissement de Bazouges, entre l'an mil et la mort de Geoffroy Martel en 1060. Ces moments ne sont pas datés précisément : les rédacteurs n'y voyaient sans doute pas d'intérêt particulier, et n'en étaient peut-être pas capables de façon précise ${ }^{33}$. Une datation relative est toutefois réalisable : il devient dès lors possible de reconstituer et de dater, par approximation, les enchaînements d'une histoire par ailleurs assez complexe.

\section{Les moines de Saint-Aubin reçoivent la cour de Bazouges (vers 974-1001)}

Cette histoire débute, nous le savons déjà, entre 974 et 1001 : Foulque Nerra remet la cour de Bazouges aux moines de Saint-Aubin ${ }^{34}$. L'intérêt réside dans la forme de cette remise : «Il donna immédiatement la moitié de cette cour avec toute l'église; l'autre moitié il la retint à lui sa vie durant seulement, selon l'accord qu'après sa mort elle soit abandonnée au dominium des moines avec toutes les améliorations [intervenues entretemps $]^{35}$. " En garantissant au comte l'exercice du dominium sur une par-

32. Voir n. 6 pour sa datation.

33. La charte $\mathrm{n}^{\circ} 1$ du cartulaire décrit ainsi des faits vieux d'un siècle et demi environ.

34. BiENVENu, Jean-Marc, L'église prieurale Saint-Jean-Baptiste de Château-Gontier, ChâteauGontier, 1964, se veut plus précis : " vers 997-998, le comte d'Anjou Foulque Nerra fit appel aux moines bénédictins de la grande abbaye Saint-Aubin d'Angers... ", mais il ne donne pas ses sources (il s'appuie sans doute sur une adresse de l'Assemblée Nationale en 1790, mentionnée par GAUCHET, R., L'église de Saint-Jean-Baptiste, Château-Gontier, 1922, p. 3, donnant la date de 998 pour fondation de l'église prieurale); Cf. C. Saint-Aubin : « [...] curte quae vocatur Basilica, quam idem ante plurimos annos proquadam curte quae nuncupatur Undanis-Villa..., monachis Sancti Albini commutaverat, eisque solidam et quietem eum omnibus ad ipsum pertinentibus ». Voir n. 6 pour sa datation.

35. C Saint-Aubin, 677 : « sed hujus curtis medietatem statim cum ecclesia tota dedit; alteran vero medietatem in vita sua sibi retinuit, ea convenientia ut, post obitum suum, cum omni melioratione, in dominium cederet monachorum ". 
tie de la cour, sa vie durant, cet accord lui ménageait une bien grande marge de manœuvre : la charte nous apprend d'ailleurs immédiatement que Foulque " donna à ses chevaliers la moitié de la cour, qu'il avait déjà donnée aux moines et dont il avait transféré la possession après sa mort $"^{36}$. Ces précisions sont importantes car elles séparent nettement les deux objets de litige entre le comte et les moines : celui concernant la partie de la cour remise immédiatement aux religieux, et celui concernant la partie que le comte doit remettre à sa mort. L'observation attentive de la charte $\mathrm{n}^{\circ} 677$ montre qu'elle n'évoque que cette dernière partie, tandis que la charte $n^{\circ} 1$ n'évoque, à notre sens, que la partie immédiatement donnée aux moines.

\section{La création du château par Foulque Nerra et sa remise à Gontier (vers 1007)}

Si nous nous tournons vers cette charte, nous voyons en effet que vers $1007^{37}$, "Foulque établit un château sur la Mayenne dans la cour de Bazouges... il l'appela Château-Gontier, du nom d'un de ses villici ". Ce villicus, c'est Gontier, qui donne son nom au lieu. L'hypothèse la plus simple voudrait qu'il se soit installé sur la moitié de cour encore sous le dominium comtal, avec pour mission d'en assurer la garde. Nous préférons toutefois suggérer qu'il le fut sur les terres déjà sous le dominium des moines. Pourquoi? Parce que nous apprenons ensuite que le comte « vit bien que l'aumône faite à Saint-Aubin... en pâtissait beaucoup. Aussi prit-il le conseil de ses proceres et décida-t-il, puisque tout le château se trouvait dans la propriété de Saint-Aubin, que ses moines en auraient le cens et les ventes $^{38}$ ". Le conseil des proceres s'avère favorable aux moines, car il fait nettement ressortir que le château s'élève sur la propriété relevant de leur droit. Le terme de dominium n'apparaît pas ici mais la remise du cens aux moines ne laisse pas de doute sur le statut de cet emplacement et sur la possession de Saint-Aubin.

36. C Saint-Aubin, 677 : "Processu vero temporis, sue ipse sponsionis et confirmationis atque excommunicationis vel oblitus vel negligens, medietatem curtis quam Sancto Albino jam condonaverat, et, post suum decessum a monachis possidendam delegaverat... suis dedit militibus ». La suite de la charte confirme qu'il est bien question ici de la partie réservée par Foulque sa vie durant.

37. Nous conservons cette datation, reprenant à son sujet les remarques de PECHA, M., "La notice $\mathrm{n}^{\circ} 1 . .$. ", art. cit., p. 6-9.

38. C Saint-Aubin, 1 : « anno ab incarnatione Dei 1007... Fulco, nobilissimus comes Andegavorum... firmavit castellum super Meduanam fluvium in curte quae vocatur Basilicas...Firmato itaque castello eoque ut poterat, ex nomine cujusdam villici sui illud castrum Gunterii appellavit. Sed quia elemosine..., dampnum maximum intulisse videbatur, cum consilio procerum suorum statuit ut, sicut totum illud castrum in proprio jure Sancti Albini consisterit, ita de toto castro monachi ejusdem Sancti census et vendas habeant et sine ulla contradictione omni tempore quiete ac libere possideant ". 


\section{L'installation de Renaud de Château-Gontier et l'édification de la tour comtale (vers 1016)}

La suite de l'acte confirme cette situation puisqu'à la faveur d'un réaménagement de ce château, Foulque Nerra y place un autre de ses milites, Renaud fils d'Yvon : il est à nouveau bien précisé qu'il versa le cens aux moines pour les biens qu'il tenait d'eux ${ }^{39}$. Ce passage donne quelques précisions complémentaires sur l'arrivée de Renaud : c'est "longtemps après " la remise du château à Gontier que « le comte entreprit d'édifier à grand frais une tour en ce château. Elle commençait de s'élever lorsqu'il eut à soutenir des guerres. Il donna alors le château, par largesse princière, à un excellent chevalier, Renaud, fils d'Yves, qui poursuit l'élévation de la tour et qui, sur ordre du comte, en versa le cens à Saint-Aubin ". Sans entrer dans la discussion relative à l'identification de ce Renaud ${ }^{40}$, il nous faut en revanche évaluer précisément le moment de son installation près de Bazouges. Un acte comtal en faveur de Saint-Florent de Saumur mentionne Renaud en 1026, en le qualifiant " de Château-Gontier " : son implantation locale est donc antérieure à cette année, et doit se situer dans ce contexte de "guerres " évoquée par la charte citée plus haut. Avant 1026, Foulque Nerra traverse deux périodes guerrières particulièrement intenses : la première en 1016 (avec la reprise des hostilités contre Eudes de Blois, qui s'achèvent par la bataille de Pontlevoy ${ }^{41}$ ), la seconde à partir de 1025-1026 (qui aboutit à la prise de Saumur ${ }^{42}$ ). Si l'on suppose que l'édification de la tour comtale n'a certainement pas été repoussée trop longtemps après l'installation du premier castrum, on retiendra plutôt la première date, c'està-dire vraisemblablement vers l'année 1016.

\section{L'hommage de Renaud à Saint-Aubin (entre 1036 et 1040)}

Renaud arrive donc à Château-Gontier dans des conditions très particulières, et finalement assez peu favorables : non seulement il doit reconnaître la seigneurie des moines, en leur versant le cens, mais en outre il doit assurer la construction d'une tour qui échappe à sa domination. L'acte est très clair à ce propos : le comte, " en homme avisé, garda la tour en sa seigneurie propre ». En homme avisé, parce qu'il évite ainsi de donner au gardien de sa tour l'élément indispensable qui lui aurait permis de s'intituler dominus : la domination sur le château ${ }^{43}$. Bien avisé, peut-être également,

39. C Saint-Aubin, 1 : le comte « dedit castrum cuidam optimo militi, Rainaldo videlicet Yvonis... de qua censum, jubente comite Sancto Albino quoad vixit temporis, libentissime reddidit".

40. AnGot, Alphonse, Généalogies féodales de la Mayenne du Xe au XII siècle, Laval, 1942, p. 137-138 et 140-144 (il suggère son appartenance à la famille de Bellême) et discussion par Gullot, Olivier, Le comte d'Anjou..., op. cit., p. 329, n. 224.

41. HALPHEN, Louis, Le comté d'Anjou..., op. cit., p. 33-35.

42. Ibidem, p. 38-40.

43. Sur ce point Gulllot, Olivier, Le comte d'Anjou..., op. cit., p. 295-296 : il évoque un cas comparable bien documenté à Saumur. 
parce que le comte déléguait à Renaud l'achèvement des travaux de la tour! Les revenus associés à la remise du château, spécialement les prérogatives banales que l'on devine détenues au nom du comte, y suffisaient-ils? La réponse semble négative puisque la suite de l'acte montre que « Renaud... avait trop peu à l'intérieur du château et, au dehors, presque rien. Aussi demanda-t-il à Gautier, alors abbé de Saint-Aubin, de lui donner une part de la cour susdite, par miséricorde. Mais rien ne put fléchir l'abbé Gautier : il refusait son accord. Enfin il céda à la demande du comte et de son fils Geoffroy Martel, et avec le conseil de la communauté il lui donne le quart de la cour de Bazouges. " Nous nous situons là entre 1036 et 1040, après l'élévation de Gautier à l'abbatiat de Saint-Aubin et avant la mort de Foulque Nerra : Renaud dispose de Château-Gontier depuis près de vingt ans et sa demande est certainement déjà ancienne. Sa situation, visiblement peu favorable, répondait-elle à un calcul de Foulque Nerra? L'hypothèse semble fort probable : en générant une rivalité entre les protagonistes castrogontériens il pouvait en effet habilement imposer sa médiation entre eux... Nous le voyons ainsi encadrer de son autorité les empiètements sur la curtis des moines, et finalement, s'entremettre entre Renaud et l'abbé Gontier pour exiger du second qu'il cède un quart de sa cour au premier. Sous la pression comtale, les moines parviennent à garantir l'essentiel : Renaud tiendra d'eux ce quart de cour, il leur versera le cens et surtout, il leur remettra son hommage tout en les assurant de sa fidélité. Les moines obtiennent ensuite, dans une formule qui leur permet de rappeler que leur dominium s'applique à l'intégralité de la curtis, que Renaud " préserve loyalement et défende pour cette église les trois quarts de la cour, de tout son pouvoir, comme un homme fidèle et un ami ». Formule d'amitié toute faite? Parions qu'en réclamant de Renaud un comportement amical, elle vient incidemment révéler une attitude préalablement hostile ${ }^{44}$... Elle suggère aussi l'habilité diplomatique des moines qui, tout en faisant reconnaître leur dominium, tempèrent ce rapport de force défavorable à leur ombrageux voisin par un lien d'amitié également partagé. Elle montre enfin leur attitude conciliante : ils misent sur des relations apaisées, quitte à perdre une partie des revenus de leur cour. Leur calcul fit ses preuves à long terme et perdura jusqu'en $1360^{45}$; à plus court terme, les moines durent vigoureusement le défendre, dans un contexte nouveau : celui de l'apparition de la châtellenie castrogontérienne.

\section{La reprise de la querelle à la mort de Foulque Nerra (entre 1040 et 1047 environ)}

Vers 1040, la situation semble arrêtée, du moins pour ce qui concerne la moitié de la cour déjà remise aux moines. Mais il en restait une autre...

44. Sur les formules et les comportements d'amitié, voir BARTHELEMY, Dominique, La société dans le comté de Vendôme..., op. cit., p. 367 et surtout p. 555.

45. En 1360 les moines échangent "cens, rentes, féages et seigneurie et tous les droits qu'ils ont dans la ville de Château-Gontier " contre diverses propriétés extérieures : arch. dép. de Mayenne, $\mathrm{H}$ 147, f ${ }^{\circ}$ 39-46. 
qui allait provoquer de nouvelles péripéties. À ce point, nous retrouvons le récit de l'acte $n^{\circ} 677$, qui indique, à première vue sans explication particulière, que les moines " déposèrent une querelle auprès du comte " contre l'injustice faite à leur encontre par son père. Le comte alors évoqué est Geoffroy Martel : le texte prend soin de préciser qu'il avait hérité de l'honor de son père, Foulque Nerra ${ }^{46}$. L'insistance des moines à évoquer la transition comtale constitue sans doute pour eux un moyen de mettre en évidence la cause du problème qui se pose alors : en 1040, à la mort de Foulque Nerra, la moitié de la cour qui devait leur être remise ne l'a pas été...

\section{Geoffroy Martel dispose d'une partie de la cour (entre 1047 et 1056 environ)}

La preuve en est immédiatement donnée, puisqu'au moment où les moines produisent une charte afin de montrer leur droit, Geoffroy Martel fait la sourde oreille et procède à un nouveau partage de cette moitié de cour. Il la remet à certains de ses fidèles, parmi lesquels les moines désignent " Eudes, comte de Bretagne ${ }^{47}$ ". Il s'agit de l'oncle et tuteur du comte de Rennes Conan II. La révolte de ce dernier en 1047 contraint Eudes à l'exil. Il se réfugie alors en Anjou, auprès de Geoffroy Martel, auquel il aurait donné une fille en mariage ${ }^{48}$. Eudes ne reçoit pas l'intégralité de la moitié de la cour puisque le comte « distribua à d'autres les autres [parties de la cour] selon son plaisir ". Ceux-là ne sont pas nommés, mais il est possible de voir en Otbert, personnage qui apparaît dans la charte $n^{\circ} 676$, un de ces " autres " disposant d'une part de la cour : Otbert fait remise de sa partie aux moines de Saint-Aubin vers 1056-1060 ${ }^{49}$, en même temps d'ailleurs qu'Eudes, dans des conditions qu'il faut expliquer.

46. C Saint-Aubin, 677 : «Postea, comite defuncto Fulcone, cum filio ejus, comes scilicet Gausfredus, potestatem paterni suscepisset honoris, querelam apud eum monachi facte sibi a patre ejus injusticie deposuerunt".

47. C Saint-Aubin, 677 : "denique etiam ipse partem medietatis ipsius curtis, que Sancto remanere debuerat Albino, comite Britannie Eudonis nomine dedit, et alias aliis sicut ejus placuit violentie, distribuit".

48. BRUNTERC'H, Jean-Pierre, " Geoffroy Martel, Conan II et les comtes bretons Eudes et Hoël de 1055 à 1060 ", dans Mondes de l'Ouest et villes du monde. Regards sur les sociétés médiévales. Mélanges en l'honneur d'André Chédeville, textes réunis par LAURENT, Catherine, MERdRIGNAC, Bernard, et PICHOT, Daniel, Rennes, 2001, p. 311-324. La gratification faite à Eudes s'explique aussi par le contexte diplomatique, alors défavorable au comte d'Anjou. Vers 1046, les agissements de l'évêque Gervais, conduisent Hugues IV du Maine à épouser Berthe, fille du comte Eudes II de Blois, et veuve d'Alain III de Bretagne : ce mariage scelle l'isolement diplomatique du comte d'Anjou, qui avait donc tout intérêt à soigner ses fidélités locales (voir GulLLot, Olivier, Le comte d'Anjou..., op. cit., p. 66-67).

49. Arthur Bertrand de Broussillon date cet acte des années 1040-1060 par l'autorisation donnée par Geoffroy Martel. Sans entrer dans le détail de l'acte, qui recoupe l'acte suivant, lui-même daté de 1056-1060, on notera la présence, parmi les témoins, des deux neveux de Geoffroy Martel, qui sont associés au gouvernement comtal à partir de 1056. 


\section{Une partie de la cour restituée ou donnée aux moines (entre 1056 et 1060)}

Nous apprenons que « le temps passant, les moines de Saint-Aubin vidèrent [la querelle?] tant par prix que par raison, avec le comte Eudes pour qu'il restitue la terre qui leur avait été injustement enlevée ". L'acte sousentend ici que la transaction fut sonnante et trébuchante, quoique les moines s'empressent de préciser qu'Eudes entend surtout réaliser la donation de cette terre. Les motivations religieuses à la remise de la terre nous semblent à cet égard assez déterminantes, et cela à plusieurs niveaux.

Dans le cas d'Otbert, la remise prend explicitement la forme d'une donation, à laquelle l'acte prévoit la contrepartie promise par les moines : ils prendront en charge l'inhumation d'Otbert, " au monastère " de Saint-Aubin, où ils feront à sa dépouille " de très honorables " funérailles. Au-delà de sa fonction funéraire, la donation permet aussi d'éteindre l'injustice et de dépasser le contentieux qui oppose les religieux à Otbert, en instituant entre eux une fraternité d'ordre religieux ${ }^{50}$.

La portée religieuse de l'acte éclaire aussi l'attitude du comte d'Anjou. La remise d'Otbert comme celle d'Eudes engage en effet l'indispensable approbation de Geoffroy Martel. Eudes précise qu'il " promit que [la remise] sera faite... à la condition que le comte Geoffroy, de qui il tenait, approuve $^{51}$ ". Immédiatement le rédacteur enchaîne : "Celui-ci approuva, par un engagement volontaire et public et donna cette terre. " Pourquoi, vers 1056-1060, Geoffroy Martel permit-il aux moines de Saint-Aubin de rentrer en possession de droit qu'il leur refusait jusque-là? C'est vraisemblablement là encore un contexte religieux qui détermine l'attitude du comte. Celui-ci cherche alors à donner plusieurs gages de bonne volonté à l'Église, d'une part en tournant la page de ses griefs contre Gervais, ancien évêque du Mans (1055), d'autre part en témoignant diverses faveurs à l'égard du monachisme pré-grégorien (1055 et 1056). Son attitude s'inscrit sans doute dans le sillage des synodes réformateurs de Lisieux et de Tours, tenus en 1054, et cherche visiblement à établir une entente solide avec le pape Victor II ${ }^{52}$. Sa bonne volonté envers les moines de Saint-Aubin pourrait bien s'inscrire dans ce cadre diplomatique : en 1056, un moine venu du milieu réformateur de Marmoutier est élu abbé de Saint-Aubin... et témoigne dans l'acte de " restitution " d'Eudes.

Le moment est donc favorable aux religieux de Saint-Aubin : la moitié de cour qu'ils auraient due recevoir à la mort de Foulque Nerra leur est si

50. C Saint-Aubin 676 : "Qua de re Gauzfredo comite auctorizante donum super altare Sancte Albini posuit, tali conventione ut, si moriatur infra viginti leugas a civitate Andecavis, aportetur ad monestrium cum processione et ibi ejus obsequium honestissime procuretur". Sur la portée du don et du contre-don : BARTHELEMY, Dominique, La société dans le comté de Vendôme, p. 422-439, et IOGNA-PRAT, Dominique, Ordonner et exclure. Cluny et la société chrétienne face à l'hérésie, au judaïsme et à l'Islam (1000-1150), Paris, 1998, p. 212-215.

51. C Saint-Aubin 677; on remarquera l'usage du verbe annuere plutôt que concedere.

52. Gulllot, Olivier, Le comte d'Anjou..., op. cit., p. 91-95. 
bien garantie que la terre remise est " totalement libre de toute domination et de coutume, et [le comte] ne retint rien pour lui dans celle-ci ${ }^{53}$ ". La référence à la dominatio établit la possession éminente des moines, et exclut par la même occasion toutes les prétentions éventuelles de celui qui aurait en garde la tour comtale de Château-Gontier. On notera d'ailleurs qu'Alard de Château-Gontier, le fils de Renaud, n'intervient à aucun moment de la transaction.

\section{Les relations du seigneur châtelain et des moines (deuxième moitié du $\mathrm{XI}^{\mathrm{e}}$ siècle)}

\section{De la cour de Bazouges à l'obédience de Saint-Jean-Baptiste}

La mort de Geoffroy Martel allait pourtant faire réapparaître au premier plan le gardien de la tour comtale de Château-Gontier. Face à lui, les moines sont conduits à consolider leur établissement. Deux problèmes chronologiques se posent à cet égard : à quel moment construisent-ils leur église et à quel moment leur établissement est-il explicitement reconnu et désigné?

La première question est la plus ardue car les propositions de datation sont aussi nombreuses que variées, et oscillent entre la fin du $\mathrm{X}^{\mathrm{e}}$ et la fin du $\mathrm{XI}^{\mathrm{e}}$ siècle ${ }^{54}$ ! Une approche archéologique récente offre toutefois une datation probable : elle situe l'édification de l'église prieurale à partir des années 1060 , voire dans le troisième quart du XI ${ }^{\mathrm{e}}$ siècle ${ }^{55}$. Cette datation est historiquement vraisemblable dans la mesure où les spoliations subies par les moines dans la première moitié du siècle rendaient difficiles la construction d'un édifice aussi monumental que l'église Saint-Jean-Baptiste (56 mètres d'est en ouest, 23 mètres du nord au sud!). On peut penser que les moines ont probablement d'abord dû utiliser l'église de Bazouges : ce n'est qu'après l'hommage de Renaud de Château-Gontier, une fois leur seigneurie consolidée, qu'ils ont dû entreprendre la construction de leur propre église ${ }^{56}$. La monumentalité de cette construction, face au château

53. On ne peut être aussi catégorique que DAVY, Christian, Les peintures murales des églises romanes du Bas-Maine, Laval, 2000, p. 245 qui, évoquant l'église Saint-Jean-Baptiste, affirme « la récupération de la totalité de la villa de Bazouges par l'abbaye de Saint-Aubin entre 1056 et 1060 ». Certaines parties sont encore remises en 1112 (C Saint-Aubin, 680).

54. Gauchet, R., L'église de Saint-Jean-Baptiste, op. cit., p. 4 (" commencé dès la fin du $\mathrm{X}^{\mathrm{e}}$ siècle, c'est-à-dire avant la fondation de la citadelle de Foulque Nerra, conçu d'un seul jet et construit sans notable interruption, le monument était complet probablement à la mort du donateur le 21 juin 1040 "); même estimation chez BIENVENU, Jean-Marc, L'église prieurale Saint-Jean-Baptiste de Château-Gontier, op. cit., p. 1-2 (dès 998, il situe en 1009 le décintrage des arcades d'après une inscription mentionnée dans un acte du XVIII ${ }^{\mathrm{e}}$ siècle, Arch. dép. de Mayenne H 15); Deyres, M., Le Maine roman, Le Mans, 1985, p. 631 et p. 381382 (achèvement vers 1020-1030); BARRAL I ALTET, Xavier, « Les peintures murales romanes de Saint-Jean-Baptiste de Château-Gontier ", 303, 22, p. 9 (dernières décennies du XI ${ }^{\mathrm{e}}$ siècle).

55. MALlet, J., Le paysage monumental de la France de l'an mil, Paris, 1978, p. 465-466, repris par DAVY, Christian, Les peintures murales..., op. cit., p. 245.

56 . Voir à ce titre la remarque pleine de bon sens de BrossaY, Charles du, "La fondation de Château-Gontier ", Province du Maine, 8, 1900, p. 201. 
de Renaud, constitue sans nul doute une affirmation de leur pouvoir. Toujours est-il que l'église Saint-Jean-Baptiste apparaît en 1076, au moment où l'évêque d'Angers Eusèbe reconnaît la possession des " autels " par SaintAubin ${ }^{57}$. Le vocabulaire épiscopal est à relever : le terme d'autel permet à la fois d'insister sur la part non appropriable de l'église et de prévenir la fondation d'une paroisse attachée au château ${ }^{58}$. De façon significative un prêtre est ainsi explicitement désigné entre 1082 et 1098, au moment même où la désignation de l'établissement monastique local se précise elle aussi ${ }^{59}$.

C'est à l'occasion d'un litige sur un étang de Château-Gontier que ces deux mentions apparaissent simultanément. Ce litige est intéressant dans la mesure où il nous permet de voir surgir l'établissement monastique dans chacune de ses composantes. Cet acte est le premier à nous montrer effectivement la présence locale des moines, ainsi que leur activité essentielle : ils sont ici pour servir Dieu et saint Jean-Baptiste ${ }^{60}$. À la désignation antérieure de la curtis de Bazouges, dont la portée strictement temporelle ne fait guère de doute ${ }^{61}$, succède ainsi une désignation dont la portée spirituelle suggère qu'une étape a été franchie dans la constitution de l'établissement local. Les longues péripéties entourant le maintien local des moines ont sans doute freiné ce passage : on notera qu'il se réalise au moment de la reconnaissance d'un modus vivendi avec le châtelain et de la construction d'une église consacrée à l'usage monastique. On ne sera dès lors pas surpris de voir apparaître simultanément les édifices abritant la communauté locale ainsi que le responsable de cette communauté : c'est "Guy de Mathefelon, qui était alors le prieur de cette obédience ", qui règle le litige " dans le cloître des moines ". Combien de religieux vivaient sous sa responsabilité? Il est difficile de l'évaluer : la liste des témoins relatifs au litige avec Garin mentionne deux moines qui pourraient être ses socii, mais ils n'apparaissent nulle part ailleurs et ne font peut-

57. C. Saint-Aubin, 19 (Bazouges n'est pas citée).

58. Sur le débat terminologique à propos des termes d'ecclesia et d'altare, confronter LEMARIGNIER, Jean-François, " le monachisme et l'encadrement religieux des campagnes du royaume de France situées au nord de la Loire, de la fin du Xe à la fin du XI ${ }^{\mathrm{e}}$ siècle ", dans Il monachesimo e la riforma ecclesiastica (1049-1122), Milan, 1968, p. 362 et BARTHELEMY, Dominique, La société dans le comté de Vendôme..., op. cit., p. 469.

59. C Saint-Aubin, 678 : "Roberto, presbytero de Sancto-Johanne "; simultanément apparaît "Roberto, presbytero de Basilgis ". Sa présence pose le problème du moment de l'érection de la paroisse de Bazouges : elle est antérieure à 1152, puisqu'à cette date une bulle du pape Eugène III confirme à Saint-Aubin, avec peu de précision, "Bazouges et ChâteauGontier ". Auparavant, le pape Urbain II avait déjà évoqué "Basilgius ", mais le terme semble se réfèrer aux «biens " (bona) de Saint-Aubin : Saint-Jean-Baptiste n'apparaît pas, sans doute parce que l'acte sous-entend son appartenance à la cour de Bazouges, qui est en fait évoquée ici. L'église Saint-Jean-Baptiste est quant à elle confirmée par le pape Pascal II en 1101 (C Saint-Aubin, 422).

60. C Saint-Aubin, 678 : « [...] Deo et sancti Albini monachis, in eodem castro Deo et sancto Johanni Baptiste servientibus ".

61. De façon significative, quand Alard de Château-Gontier renouvelle sa reconnaissance de l'immunité possédée par les moines sur ses coutumes, en 1109, il évoque la " curtem dominicam quam habent monachi... que basiligia dicitur " 
être qu'accompagner l'abbé Girard. La présence de l'abbé lors du règlement de ce litige est à relever : elle souligne le lien étroit qui unit l'obédience et son abbaye. Ce lien perdure très visiblement tout au long du $\mathrm{XII}^{\mathrm{e}}$ siècle $^{62}$ : il limite sans doute l'autonomie de l'établissement local et explique pourquoi nous ne connaissons les noms que de trois prieurs pour toute cette période.

Cette dernière remarque ne nous empêche toutefois pas d'y voir un peu plus clair. Une rapide récapitulation de nos observations montre que les éléments qui structurent l'identité du prieuré sont présents dès la fin du $\mathrm{XI}^{\mathrm{e}}$ siècle : le prieur dispose d'une position éminente, en relation étroite avec l'abbé, et dirige ses compagnons dans un logis installé près de l'église Saint-Jean-Baptiste. Cet édifice imposant révèle avec ostentation la présence sacrée des religieux face à la tour du seigneur local : il marque symboliquement leur affermissement. Mais pouvait-il suffire à lui seul à garantir la position des moines face au châtelain? Évidemment pas!

\section{Les moines de Saint-Aubin et leurs soutiens face à la seigneurie châtelaine}

Dans leur face-à-face menaçant avec le seigneur de Château-Gontier, les religieux devaient en effet également se ménager de solides soutiens locaux. À cet égard, les relations avec les prêtres de leurs églises de ChâteauGontier et Bazouges leurs permettent sans doute de trouver un relais influent auprès des populations locales ${ }^{63}$. Il est évident qu'ils sollicitèrent également la société aristocratique locale : malgré une documentation lacunaire en la matière, quelques indices nous laissent en effet entrevoir les relations qu'ils entendent privilégier. La famille de Mathefelon semble ici jouer un rôle déterminant, au point de donner au prieuré son premier prieur connu ${ }^{64} \ldots$

Installés après 1028 par Foulque Nerra à Cheffes, au sud de ChâteauGontier, afin d'y défendre les possessions de l'abbaye du Ronceray, les Mathefelon disposent de larges possessions autour de Château-Gontier. Ces

62. Il faut attendre 1193 pour découvrir un acte sans référence à l'intervention de l'abbé de Saint-Aubin (C Saint-Aubin, 686); les 10 actes antérieurs à notre disposition montrent tous son intervention. Rappelons que le terme d'obédience est généralement utilisé de manière à souligner la dépendance de l'établissement vis à vis de son abbaye mère (BAUTIER, Anne-Marie, "De "prepositus" à "prior"... ", art. cit., p. 15 sqq). A titre de comparaison, si le terme d'obédience est le qualificatif exclusif désignant un établissement de Marmoutier dans le Bas-Maine jusqu'à la fin du XI ${ }^{\mathrm{e}}$ siècle, il disparaît au XII ${ }^{\mathrm{e}}$ siècle alors que les établissements gagnent en autonomie : l'abbé de Marmoutier n'intervient ainsi directement que dans 2 des 28 actes relatifs au prieuré Saint-Martin de Laval entre 1060 et 1210 (voir LEgros, Sébastien, Les moines et la société à la fin du Moyen Âge. Un cas d'étude dans le Bas-Maine : les prieurés de Marmoutier (XIV -XV siècle), mémoire de DEA, dactyl., Rennes, 2001, p. 102).

63. Les presbytres de Saint-Jean-Baptiste et de Bazouges témoignent dans le litige contre Garin (C Saint-Aubin, 678).

64. Guy de Mathefelon (Angot, Alphonse, Généalogies..., op. cit., p. 320-321). 
possessions, spécialement à Azé et à Quelaines, et de façon plus éloignée à la Cropte et à Parné, en faisaient des partenaires potentiellement utiles dans un environnement où les moines ne disposent guère de soutien. Les religieux exploitèrent habilement cette opportunité, grâce à des liens dont on peut penser qu'ils apparaissent vers le milieu du XI ${ }^{\mathrm{e}}$ siècle. Les origines locales de l'abbé Girard jouèrent sans doute en la matière un rôle impor$\operatorname{tant}^{65}$ : en 1049, Girard témoigne en effet en compagnie d'Hugues Mansel et son frère homonyme Hugues, lors d'une donation du comte Geoffroy Martel en faveur de la Trinité de Vendôme ${ }^{66}$. Hugues Mansel est le fondateur de la lignée des Mathefelon, mais c'est son frère qui nous intéresse spécialement. Hugues de Mathefelon fit en effet profession monastique auprès de SaintAubin, avant la date de 1087 : nous le voyons paraître à cette époque dans diverses donations, notamment lors des fondations, vraisemblablement conjointes, des prieurés de Quelaines et de Saint-Gault ${ }^{67}$. Il semble même diriger l'établissement de Quelaines, où il est mentionné jusque vers $1100^{68}$. En deux autres occasions, il intervient pour régler un litige en compagnie ou à la demande expresse de l'abbé Girard : on le voit intervenir auprès de Rivallon de Quelaines, fondateur du prieuré du même nom, puis en tant qu'envoyé de l'abbé Girard auprès de la Trinité de Vendôme dans un litige (de longue haleine) au sujet du prieuré Saint-Clément de Craon ${ }^{69}$. Son rôle nous semble donc déterminant dans l'établissement des liens entre les religieux de Saint-Aubin et la famille de Mathefelon, et le rapproche du cas comparable du moine Jean, fils de Guy Ir ${ }^{\text {er }}$ de Laval, artisan du rapprochement entre sa famille et l'abbaye de Marmoutier. Dans l'un et l'autre cas, l'approfondissement des relations passe par la profession d'un membre éminent de la famille aristocratique concernée, appelé à exercer d'importantes responsabilités locales en faveur de son abbaye ${ }^{70}$.

L'utilité de telles relations est évidente pour les moines de ChâteauGontier qui gagnent un soutien aristocratique d'importance en cas de litige... et pour les Mathefelon qui savent aussi solliciter l'appui des moines à leur avantage. De fait, si Foulque de Mathefelon, le fils de Hugues Mansel,

65. Girard devient abbé de Saint-Aubin en 1082; Angot, Alphonse, Dictionnaire..., op. cit., t. 2, p. 297 ainsi que CHAMARD, D., Les saints personnages de l'Anjou, t. 2, 1869, p. 158204 (spécialement p. 158, n. 1, pour une présentation succincte des sources) : Girard serait né près de Bazouges, au lieu de l'Oisellière. Il aurait été presbytre de Bazouges avant de faire sa profession puis de devenir abbé de Saint-Aubin.

66. C. Trinité de Vendôme 92 : il est alors presbytre.

67. C Saint-Aubin, 900 : "Audientibus et his : Hugone Mansello, Sancti Albini monacho, qui tunc erat temporis ecclesie illius preerat."

68. C Saint-Aubin, 905 : «Hubertus Blineus ingagiavit medietatem terre cum prato adjaente, apud Colonias, Hugoni Mansello, monacho Sancti Albini aciipiens pro ea quindecim solidos cenomannensium denariorum. "

69. C Saint-Aubin, 900, 903 (avec Rivallon de Quelaines), 905; C TV, 346.

70. Nous nous permettons de renvoyer à LEGROS, Sébastien, "Le prieuré Saint-Martin de Laval et la consolidation de la seigneurie lavalloise au $\mathrm{XI}^{\mathrm{e}}$ siècle ", dans La Mayenne Archéologie Histoire, 26, 2003, p. 12-43. Sur l'importance des conversions à la vie monastique au milieu du XI ${ }^{\mathrm{e}}$ siècle : MAZEL, Florian, La noblesse et l'Église en Provence, fin $X^{e}$-début XIV siècle, Paris, 2002, p. 176-177. 
soutient les moines dans le litige, déjà évoqué, qui les oppose à Garin, et leur donne, vers 1087, l'église de Quelaines, il profite du même coup du prestige inestimable conféré par sa familiarité avec les religieux. En remettant l'église de Quelaines à l'abbé de Saint-Aubin, il obtient en effet de Girard qu'il cautionne la position locale : en témoignant au côté de Foulque, l'abbé le gratifie d'une belle titulature qui légitime ses possessions à la Cropte, au moment même où un homme du seigneur de Laval vient les lui contester ${ }^{71}$. Les intérêts bien compris des partenaires sont ainsi renforcés par une association qui consolide leur enracinement local.

\section{Déprédations et exactions du dominus de Château-Gontier}

Si les religieux recherchent si activement de tels soutiens, c'est que la situation locale évolue doublement dans la seconde moitié du $\mathrm{XI}^{\mathrm{e}}$ siècle : d'une part en se durcissant notablement, d'autre part en se compliquant par l'apparition de nouveaux acteurs religieux.

Parmi ceux-ci les moines de la Trinité de Vendôme apparaissent vers 1040. Ils s'installent au lieu de Ménil, qui appartient à leur abbaye depuis sa fondation par Geoffroy Martel ${ }^{72}$. Les moines disposent alors de "l'église du vicus de Ménil et toute sa cour ${ }^{73}$ ". On retrouve ici l'association assez classique ecclesia-curtis, qui semble désigner l'église et son patrimoine. Le qualificatif de vicus est également intéressant compte tenu des antécédents du site ${ }^{74}$. Le cas de Ménil semble finalement assez proche de celui de Château-Gontier : même donation comtale initiale et même type de contenu (quoique de manière vraisemblablement plus limitée). Les conditions sont toutefois sensiblement différentes puisque le châtelain de Château-Gontier possède explicitement des coutumes à Ménil à partir de 1060 au plus tôt ${ }^{75}$. Celles-ci lui ont été données par Foulque Réchin, en faveur duquel Renaud II de Château-Gontier avait pris parti lors de la lutte qui l'opposait à son frère, Geoffroy le Barbu. Si nous ignorons les conditions précises qui ont présidé à la remise de ces coutumes (une gratification du comte Geoffroy en faveur d'un de ses fidèles est possible), nous en connaissons en revanche précisément les conséquences : les moines subissent rapidement l'hostilité et les violences du seigneur de Château-Gontier.

71. C Saint-Aubin, 742 (nous nous permettons à nouveau de renvoyer à une présentation détaillée de ce contexte dans LEGRos, Sébastien, " Le prieuré Saint-Martin de Laval... ", art. cit., p. 12-43).

72. Voir Gulllot, Olivier, Le comte d'Anjou..., op. cit., p. 55 sqq et Johnson, Penelope, "Pious legend and historical realities. The foundation of la Trinité de Vendôme, Bonport and Holyrood ", Revue bénédictine, t. 91, 1981, p. 184-187.

73. C TV, 38 (fondation de l'Evières, qui reprend la charte de fondation de l'abbaye ellemême) : "ecclesias vici Meduanilis et curtem totam ".

74. Voir ci-dessus, n. 16 à ce propos. La confirmation de la possession de l'église de Ménil par la Trinité, en 1056-1057 puis 1063, par les papes Victor II et Alexandre II, reprend également le qualificatif de vicus (C TV, 107).

75. C TV, 297 : Foulque Réchin explique, entre 1068 et 1078 : " consuetudinibus quas in burgo Meduanilis me tibi donasse asseris ". 
C'est une injonction du comte d'Anjou, malheureusement mal datée (entre 1068 et 1078) qui nous révèle brutalement cette hostilité. Son caractère est indubitablement seigneurial, mais elle procède peut-être aussi d'une attitude anti-grégorienne. Toujours est-il que le comte réprimande son " très cher fidèle " Renaud (III de Château-Gontier), afin qu'il cesse ses prédations; il explique que « les coutumes que je t'ai données dans le bourg de Ménil ne te permettent pas de molester en plus les moines de Vendôme, ni avec elles de chercher à les atteindre selon ton plaisir ${ }^{76}$ ". Le comte rappelle la fondation de l'abbaye de la Trinité par son oncle afin de souligner deux faits. D'une part les moines disposent de leur biens librement et paisiblement, " comme il est reconnu pour un monastère de l'alleu de SaintPierre " : la portée grégorienne de la formule fournit ici un bel argument pour le comte face à l'un de ses hommes, dont il sent vraisemblablement les turbulentes volontés d'émancipation ${ }^{77}$. D'autre part, il incombe au fondateur de protéger les églises de la Trinité, ce qui permet d'asséner une menace directe : "Si tu fais une autre injure ou inquiétude auprès des moines, ce n'est pas tant à eux qu'à moi que tu causeras un désagrément. Et c'est moi qui défend et protège ce monastère et toutes ses possessions contre tout homme. " La menace n'est pas voilée et elle montre la détérioration des relations entre Renaud et le comte. Fut-elle suivie d'effet? On peut fortement en douter, car le litige reste latent près de trente ans durant : ce n'est qu'en 1107 que le poids des forces grégoriennes parvient à faire plier celui qui, entre-temps, est devenu le dominus de Château-Gontier ${ }^{78}$. Profitant du passage du pape Pascal II, l'abbé de la Trinité obtient en effet l'appui de l'évêque d'Angers qui " excommunia ledit Alard et mit l'interdit de l'office divin sur toutes les terres de celui-ci ». Trois points doivent ici être relevés.

D'une part le règlement du litige, en 1107, laisse entièrement de côté le comte d'Anjou : son intervention (en 1068-1078) n'a visiblement pas eu d'effet. L'acte de 1107 prend d'ailleurs bien soin de souligner qu'Alard ne fait que perpétuer les prédations de son père, duquel il a hérité un caractère le portant à la violence et à l'injustice. On ne saurait mieux exprimer la durée du litige, avec le père puis avec le fils. D'autre part, il faut remarquer que le litige porte sur le bourg de Ménil et sur les coutumes qui y sont levées. La

76. C TV, 297 : " Mando tibi sicut karisimo meo fideli, et interdico ut de consuetudinibus quas in burgo Meduanilis me tibi donasse asseris, ulterius monachos Vindocinenses molestare non presumas, neque cum eis super hoc placitari contendas, quod nec tibi dedi nec dare potui".

77. Alors même que sa propre réputation auprès de la Trinité n'était pas sans tâches (Johnson, Penelope, Prière, patronage et pouvoir. L'abbaye de la Trinité de Vendôme, 10321187, Vendôme, 1997, p. 95-96). La remarque permet au comte de s'inscrire dans la ligne du rapprochement réalisé par son prédécesseur avec la papauté.

78. C TV, 417 : l'abbé de la Trinité explique que « les coutumes et exactions qu'Alard de Château-Gontier et Renaud son père avaient imposées par violence dans notre bourg de Ménil, touchent à leur fin. Ainsi, comme nous le disons, Alard exigeait injustement certaines coutumes et exactions sur ce bourg et sur nos hommes de ce bourg, et ce qu'il avait voulu il l'obtenait par la violence ". 
dimension seigneuriale est donc essentielle, à un moment (vers 1096-1098) où Renaud III de Château-Gontier franchit le pas de l'émancipation à l'égard du comte d'Anjou et revendique le titre de dominus ${ }^{79}$. Ce contexte nous permet de retrouver nos religieux de Saint-Aubin dans la mesure où leur propre bourg aurait alors subi, lui aussi, les déprédations du seigneur de ChâteauGontier. Deux éléments laissent supposer ce rapprochement. En premier lieu les moines angevins obtiennent, en 1109, la reconnaissance de leurs droits de seigneurie dans la " cour seigneuriale de Bazouges ${ }^{80}$ ", puis, en 1112, la remise de diverses coutumes, que le seigneur de Château-Gontier percevait indûment, " dans la terre de Saint-Aubin ", et spécialement "sur les maisons de leur bourg ". À cette occasion AlardIII, qui était malade, apparaît " regrettant... les nombreux et grands maux qu'il a causé à l'abbaye de Saint-Aubin ${ }^{81}$ ". Fait-il ici référence à des préjudices locaux? C'est évidemment probable et il y a tout lieu de croire que les troubles affectent en réalité conjointement les possessions des moines angevins et tourangeaux. On peut également penser que les moines de Saint-Aubin ont su profiter du règlement en faveur des moines de Ménil : l'intervention épiscopale et l'excommunication d'Alard leur conféraient des arguments efficaces pour résoudre leurs propres problèmes. Cette intervention de l'évêque d'Angers nous amène à une seconde observation. Le règlement du litige de Ménil fait en effet apparaître, auprès des moines de la Trinité, la présence des prêtres de Bazouges et de Saint-Jean-Baptiste. Leur présence vient évidemment sanctionner la mobilisation des clercs séculiers locaux autour de l'intervention épiscopale. Mais pourrait-elle aussi exprimer un soutien sous-jacent des religieux de Saint-Aubin, patrons de ces deux prêtres? L'hypothèse ne doit pas être écartée, même si de lourds contentieux séparent alors les deux abbayes (au sujet de Saint-Clément de Craon) : la résolution des problèmes concernant l'un des deux prieurés intéressait nécessairement l'autre, et la présence des deux prêtres liés à Saint-Aubin, si elle souligne l'unanimité des séculiers locaux derrière l'évêque, laissent aussi entrevoir l'unanimité des religieux contre les exactions du seigneur de Château-Gontier. On notera, à cet égard, que les moines de Saint-Aubin appuient leurs propres demandes par la présence, 1109 comme en 1112, de l'évêque d'Angers Renaud.

79. Voir sur ce point les analyses de GulLLot, Olivier, Le comte d'Anjou..., op. cit., p. 305309 (analyse du terme de dominus), p. 315-317 (analyse du processus d'apparition des seigneuries châtelaines : il en signale 16 nouvelles entre 1082 et 1109) et appendice 2 , p. 463 : mention de "Raginaldus castri Gunterii dominum " entre le 22 août 1096 et le 14 mars 1098 (dans Notice de Saint-Nicolas, Epitome, p. 32). Analyse du problème par PECHA, M., "La notice ${ }^{\circ} 1$... ", art. cit., p. 18-20.

80. C Saint-Aubin, 679 : «Adelardus castri Gunterii dominus, Rainaldi Jerosolimiti filius, curtem dominicam quam habent monachi Sancti Albini prope Castrum Gunterii que Basilica dicitur, immunem ab omni consutudines esse recognovit..."

81. C Saint-Aubin, 680 : "Adelardus Castri Gunterii dominus, judicio Dei infirmatus, visitatus est ab episcopo Andegavensi, Rainaldo, et abbate Sancti Albini Archembaldo. Quibus presentibus, penitens ille super multis et magnis malis que abbatie Sancti Albini intulerat, quasdam consuetudines, quas in terra Sancti Albini habebat, ejusdem Sancti in perpetuum condonavit..." 


\section{L'affermissement de la seigneurie châtelaine dans le contexte grégorien : la fondation du prieuré d'Azé face aux religieux de Saint-Aubin et à la famille de Mathefelon}

Derrière ces litiges, se dessine l'image peu favorable d'un seigneur de Château-Gontier, présenté comme un spoliateur déterminé des établissements monastiques locaux. Le portrait est évidemment forcé par le regard ecclésiastique, mais il commande de s'interroger sur le comportement du seigneur. Il s'agit en particulier de se demander si son hostilité envers les religieux est associée, d'une manière ou d'une autre, à son émancipation en tant que dominus. La question est complexe, car les seigneurs de ChâteauGontier savent visiblement bien souffler le chaud et le froid, selon leurs intérêts et selon que le contexte grégorien leur offre, ou non, des opportunités.

Les liens que Renaud III de Château-Gontier parvint ainsi à établir avec l'abbaye Saint-Nicolas sont à cet égard exemplaires, nous semble-t-il, de son habilité à user d'un soutien prieural au service de ses ambitions seigneuriales. Nous voyons en effet, entre 1080 et 1098, le dominus castrogontérien réaliser plusieurs donations en faveur des moines de Saint-Nicolas et finalement participer à la fondation du prieuré d'Azé ${ }^{82}$. Il prend soin de justifier son attitude par une longue motivation d'inspiration grégorienne où il insiste longuement, à cet égard, sur la vanité de la vie d'ici-bas et sur la nécessité, pour celui qui recherche les récompenses célestes, à se séparer de ses richesses ${ }^{83}$. Ce programme, assez original au regard des violences que Renaud pratique par ailleurs, répond, sans doute, à l'influence de sa mère Elizabeth, qui réalise elle-même une donation en faveur du prieuré d'Azé ${ }^{84}$. Mais ce beau programme cache peut-être d'autres préoccupations... plus temporelles. Nous ferons à cet égard deux commentaires.

Il est d'abord intéressant d'observer le contenu des donations de Renaud et d'Elizabeth (fig. 2) : outre des biens à Segré, leurs dons viennent

82. Brossay, Charles du, Cartulaire d'Azé et du Genéteil, prieurés de l'abbaye Saint-Nicolas d'Angers (1080-1637), Archives historiques du Maine, t. 3, Le Mans, 1903, p. 51 sqq (désormais abrégé C AG, 1, 2 et 4). Nous n'évoquerons pas ici les liens entre le seigneur de Château-Gontier et l'abbaye du Ronceray (voir le chapitre consacré à Château-Gontier par MARChegay, Paul, Cartularium monasterii Beatae Mariae Carittis andegavensis, Recueil de documents inédits sur cette province d'Anjou, Archives d'Anjou, t. 3, Angers, 1854, p. 87-90).

83. C AG, 2 : « La convoitise a traversé le monde. Celui qui est né est mort; celui qui s'est développé s'est desséché. Rien de ceci n'est joie, rien de cela n'est perfection... Ceux qui se réjouissent des dignités, ceux qui se sont élevés par le pouvoir et ceux qui se sont nourris des choses d'ici-bas, reconnaissant la vérité... ils sont plutôt appelés malheureux que saints. Et puisque les choses terrestres ne promettent ni ne donnent à voir la béatitude, moi, Renaud de Château-Gontier, apparaissant comme un humble pécheur devant Dieu et considérant que ni par cela, ni par l'aide attendue des autres hommes, mais que, averti en cela par les Ecritures, le péché pourra être racheté par les aumônes à Dieu et... ainsi trouver une grande rétribution... " Sur les préambules des actes : voir PARISSE, Michel, "Une enquête à mener : la spiritualité des nobles au miroir des préambules de leurs actes ", dans Georges Duby. L'écriture de l'histoire, Paris, 1996, p. 306-316.

84. C AG, 1 . 


\section{Carte 2 - Possessions des abbayes Saint-Aubin et Saint-Nicolas autour de Bazouges (inventaire du $\mathrm{XI}^{e}$ au $\mathrm{XV}^{e}$ siècle)}

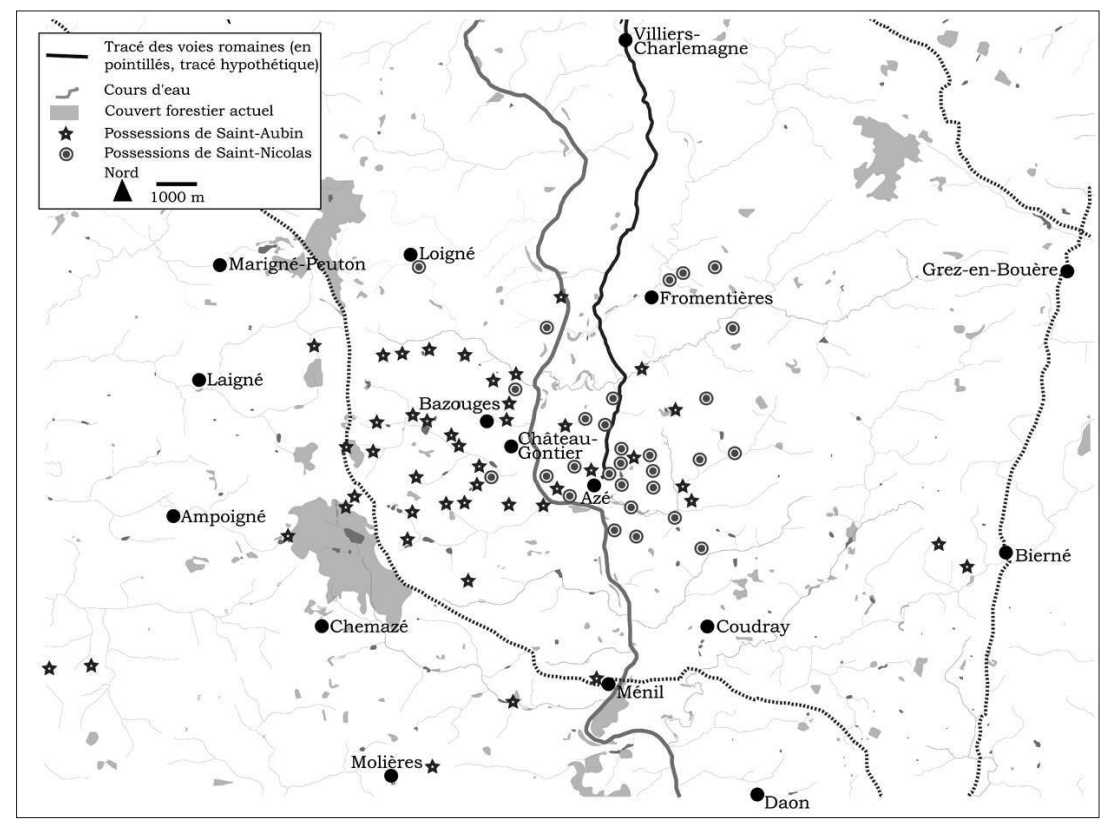

singulièrement offrir aux moines de Saint-Nicolas des possessions qui avoisinent celles du prieuré Saint-Jean-Baptiste, sur la rive droite de la Mayenne $^{85}$. On peut se demander si la distribution de ces donations initiales ne vise pas à concurrencer les moines de Saint-Aubin. Si tel est bien le cas, le résultat est mitigé : sur la rive droite de la rivière ces donations sont ponctuelles et resteront sans suite, de sorte que la rive reste " chasse gardée " des moines de Saint-Aubin ${ }^{86}$. La remarque s'inverse si l'on considère la rive gauche : ici les religieux de Saint-Nicolas dominent et empêchent l'implantation de leurs concurrents angevins ${ }^{87}$. Bref : chacun consolide ses positions de part et d'autre de la rivière et neutralise son rival.

On peut aussi s'interroger sur les raisons qui expliquent la localisation du prieuré de Saint-Nicolas à Azé. Dans un contexte grégorien le choix est surprenant, car Renaud ne possède pas l'église d'Azé, qui appartient au prêtre local, Garin le Boiteux, et à Garsias du Bignon. L'étude de ces deux

85. Près du moulin de Bressac, situé à $2 \mathrm{~km}$ à l'ouest de Ménil sur le ruisseau de Rouillard, à la métairie du Coudray, voisine de Bressac, et à Quelaines.

86. Hormis quelques clos de vignes à Vauvert, à $1 \mathrm{~km}$ au sud de Bazouges, que nous découvrons dans le patrimoine des religieux d'Azé en 1403 (C AG, 71, 87 et 89). La remarque relative à la distribution du patrimoine vaut également pour le prieuré du Genéteil, dépendant de Saint-Nicolas, fondé quant à lui en 1120.

87. Pour autant qu'on puisse les reconstituer jusqu'au $\mathrm{XV}^{\mathrm{e}}$ siècle, les possessions du prieuré d'Azé se regroupent exclusivement sur la rive gauche de la Mayenne. 
personnages permet de mettre en évidence quelques éléments tout à fait suggestifs. La famille de Garin, d'abord, semble étroitement liée à SaintNicolas. Nous savons en effet que Garin avait deux frères : Geoffroy et Morel. Un (seul) autre duo de frères prénommés Geoffroy et Morel apparaît dans notre documentation locale, précisément dans la charte $n^{\circ} 6$ du Cartulaire d'Azé et du Geneteil. Celle-ci permet, en toute hypothèse, de relier nos trois personnages à la famille du Rallay ${ }^{88}$ et d'éclairer les liens qui unissent leur famille à l'abbaye de Saint-Nicolas. Imitant son frère Garin, Geoffroy réalise en effet une importante remise de dîmes et de coutumes dans son fief en faveur des religieux d'Azé; il participe aussi activement à la fondation du prieuré de Gennes ${ }^{89}$... et achève finalement sa vie sous l'habit monastique de Saint-Nicolas ${ }^{90}$. Les moines récompensent généreusement ses attentions : son nom est inscrit au martyrologue de l'abbaye, tandis que sa femme Agnès et son fils, singulièrement prénommé Garin, sont admis dans le bénéfice des moines et obtiennent la permission d'être inhumés à l'abbaye ${ }^{91}$. Une telle politique religieuse commandait évidemment à la famille du Rallay d'obtenir les autorisations féodales nécessaires. En la matière, les choses sont relativement claires et révèlent la présence d'une famille, bien connue par ailleurs et déjà évoquée en relation avec Saint-Aubin : la famille de Mathefelon. En 1219, un certain Jehan du Rallay obtient ainsi de Thibaud de Mathefelon l'autorisation de donner une partie de son moulin de Dordogne aux religieux de Saint-Nicolas ${ }^{92}$. Le lien féodal entre les deux familles est en réalité beaucoup plus ancien et nous ramène à Garsias du Bignon, co-donateur avec Garin le prêtre de l'église d'Azé en faveur de Saint-Nicolas ${ }^{93}$. Par sa mère Béatrice, fille de Hugues Mansel, Garsias est en effet le neveu de Foulque de Mathefelon dont la famille semble anciennement enracinée à Azé : son père y détenait des dîmes qui sont remises à Saint-Nicolas vers 1100 dans un geste explicitement grégorien ${ }^{94}$.

Voilà donc que l'implantation des moines de Saint-Nicolas à Azé nous révèle l'intervention, plus ou moins directe, de la famille de Mathefelon. Comment le seigneur de Château-Gontier réagit-il à cette situation? Dans une perspective de stricte rivalité, sa position semble délicate entre les possessions de religieux de Saint-Aubin, autour de Bazouges, et celles des

88. Dans C AG 6 Geoffroy du Rallay fait une donation à Saint-Nicolas, avec l'approbation de son frère Morel; Geoffroy et Morel réapparaissent ensemble pour consentir à la donation de Garin le presbytre dans C AG 4. Cette famille possède le lieu du Rallay, à 2 km au nord-est d'Azé, où une motte a été identifiée (Rouet PaILlard de Clermont, S., Les fortifications en terre dans la baronnie de Château-Gontier, $X I^{e}-X V^{e}$ siècles, mémoire de maîtrise, Université du Maine, 1994, p. 102).

89. Angot, Alphonse, Dictionnaire..., op. cit., II, p. 278. Gennes est situé à une dizaine de kilomètres au nord-est d'Azé.

90. C AG, 6. Les seigneurs du Rallay apparaissent jusqu'en 1219 en relation étroite avec Saint-Nicolas (C AG, 6, 7, 23, 29).

91. C AG 6.

92. C AG 29.

93. Garsias fait également concession de la partie donnée par Garin.

94. Restitution de Foulque et de sa femme Hersende (C AG 5). 
Mathefelon sur la rive gauche de la Mayenne, autour d'Azé. L'impulsivité du seigneur de Château-Gontier devrait logiquement le conduire à ces gestes d'hostilité dont il est par ailleurs coutumier. Or, nous l'avons vu, il n'en est rien : alors même qu'il ne pouvait ignorer ce contexte féodal, c'est lui qui initie les gratifications locales en faveur de l'abbaye (à Azé, puis au Genéteil) et qui cherche, malgré quelques difficultés ponctuelles, à soigner ses relations avec l'abbé de Saint-Nicolas ${ }^{95}$. Sans doute cherche-t-il ainsi à compenser ses fâcheries avec Saint-Aubin et la Trinité. Sans doute veut-il aussi éviter une opposition directe avec les Mathefelon. Sans doute enfin, poursuit-il d'autres objectifs, plus habiles, afin de contrer l'influence de ses rivaux locaux.

Habiles, certes, car Renaud de Château-Gontier aura su exploiter la dévotion des Rallay en faveur de Saint-Nicolas, et la volonté du prêtre Garin de restituer sa partie de l'église locale, en l'associant à ses propres donations. Habiles ensuite, parce que dans le contexte grégorien, Renaud enclenchait ainsi un processus qui avait toute chance d'aboutir à la restitution des autres biens ecclésiastiques locaux, notamment les dîmes. De façon pratique, cela revenait à distraire ces différentes possessions du patrimoine des Mathefelon, en les attribuant aux religieux de Saint-Nicolas. Pour le dire de façon prosaïque, le prieuré d'Azé permettait au seigneur de ChâteauGontier d'amoindrir le patrimoine local d'une famille rivale, par ailleurs alliée à un prieuré rival. On le devine, les Mathefelon furent récalcitrants : vers 1100, lors de la restitution des dîmes d'Azé, Foulque de Mathefelon fit payer son approbation au prix fort de 55 livres! Dès sa mort, son fils Hugues reprit brutalement les dîmes et n'accepta de les restituer qu'après son excommunication par l'évêque d'Angers. À la même époque, c'est au tour de Garsias du Bignon de refuser aux moines le droit d'élire le prêtre d'Azé au prétexte que ce droit appartenait à ses ancêtres. Là encore il faut l'intervention de l'abbé et de l'évêque pour le faire plier et obtenir la concession de ses frères et de son fils ${ }^{96}$.

Ces difficultés et ces litiges constituaient-ils un effet recherché par la donation initiale du dominus de Château-Gontier? L'hypothèse nous semble d'autant plus séduisante qu'elle offre une piste de compréhension des modalités mises en œuvre par un dominus pour consolider sa seigneurie châtelaine naissante. Exploitant le contexte grégorien, qu'il subissait par ailleurs lui-même largement, le seigneur de Château-Gontier parvient en effet, par l'entremise du prieuré, à contrarier la famille de Mathefelon, et, à travers elle, à contenir les moines de Saint-Aubin. Ces derniers développements montrent l'importance prise par les prieurés dans le cadre de l'établissement des seigneuries châtelaines. Le cas est tout à fait net pour le prieuré d'Azé : d'un point de vue temporel, son apparition offre au seigneur

95. Sur la fondation du prieuré de Genéteil : C AG 10 et 11, vers 1121; quelques années après (vers 1129), le successeur de Renaud III, Alard II de Château-Gontier, est malade et réclame la venue de l'abbé de Saint-Nicolas pour le soigner.

96. C AG, 9 . 
de Château-Gontier l'occasion de distraire à une famille rivale des biens concurrençant localement son influence. Une attitude comparable se repère chez le seigneur de Laval, qui exploite quant à lui le réseau de prieurés mis en place par l'abbaye de Marmoutier ${ }^{97}$.

Les prieurés nous apparaissent finalement assumer un rôle contradictoire, dont il faudrait préciser la réalité à plus large échelle : doit-on voir en eux une instance originale de régulation des rivalités féodales et seigneuriales ou des acteurs nettement plus assumés des rivalités, quitte à les envenimer le cas échéant? La réponse se trouve vraisemblablement dans un entre-deux qui doit beaucoup à la spécificité des contextes locaux. Car ce qui vaut pour un prieuré ne vaut pas nécessairement de façon identique pour un autre : à cet égard, si le prieuré d'Azé profite de la bienveillance du seigneur de Château-Gontier, les établissements de Saint-Aubin et de la Trinité de Vendôme provoquent quant à eux une attitude franchement plus hostile, voire violente. Ces établissements présentent pour lui un inconvénient majeur : ils précèdent l'apparition de sa seigneurie châtelaine. Ils apparaissent donc comme des voisins encombrants parce qu'ils disposent de terres et de droits qui concurrencent ou limitent le patrimoine et les prérogatives du seigneur local. La création du prieuré Saint-Jean-Baptiste, issu de la cour comtale de Bazouges, est exemplaire de ce type de rivalité qui, il faut le souligner, ne vint pas à bout de la détermination des moines face au châtelain. La politique des religieux en la matière vise, avec constance, à assurer et défendre la pérennité de leur établissement et de leur dominium $^{98}$. Dans ce cadre, ils assument parfaitement les impératifs du face à face social qui définit leur époque. Ils passent de l'hostilité et de la lutte à la recherche des concessions qui pourront, si cela leur convient, limiter les gestes d'hostilité et créer les conditions d'une amitié : nous l'avons constaté à l'endroit de Renaud I Ir de Château-Gontier. Ils savent aussi parfaitement user des rivalités seigneuriales, ce qui explique les liens qu'ils tissent avec la famille de Mathefelon. Ils utilisent enfin avec habilité les armes que leur procure le courant grégorien, usant systématiquement de l'appui épiscopal, et menaçant régulièrement de recourir à l'excommunication. Leur attitude fut avantageuse, même si elle se paya au prix de l'indifférence absolue du seigneur de Château-Gontier, qui, fait remarquable, ne leur adressa pas la moindre donation. Elle permit en tout cas l'affermissement conjoint d'une seigneurie châtelaine et surtout d'un prieuré qui nous laisse une documentation volumineuse, témoin de sa prospérité jusqu'à la fin du Moyen Âge.

97. Nous nous permettons de renvoyer ici à LEGROS, Sébastien, « Le prieuré Saint-Martin de Laval... ", art. cit.

98. MÉHu, Didier, Paix et communauté autour de l'abbaye de Cluny, Lyon, 2001, p. 230-236. 


\section{RÉSUMÉ}

Cet article s'attache à retracer le lien qui unit la fondation du prieuré Saint-Jean-Baptiste de Château-Gontier et l'émancipation châtelaine du seigneur de ce lieu. Elle met en lumière deux périodes au $\mathrm{XI}^{\mathrm{e}}$ siècle. La première moitié du siècle est marquée par l'emprise du comte d'Anjou, donateur initial de la curtis de Bazouges aux moines et à son villicus Gontier : entre 1001 et 1060, le comte manœuvre afin de préserver sa mainmise, en orchestrant l'opposition des moines et du châtelain... puis en imposant sa médiation entre eux. Dans la seconde moitié du siècle, la mort de Geoffroy Martel marque l'affaiblissement du contrôle comtal et renforce l'opposition entre les moines et le dominus de Château-Gontier. Les moines placent la dispute sur le terrain grégorien et appuient leur position sur leur amitié avec les Mathefelon, rivaux locaux du seigneur. La riposte du seigneur consolide sa position : en contrepoids aux religieux de Saint-Aubin, il implante à Azé un prieuré concurrent, dépendant de Saint-Nicolas. Les prieurés auront joué un rôle essentiel dans l'émancipation du dominus de Château-Gontier.

\section{ABSTRACT}

The purpose of this study is to examine the link which bonds the fondation of the Saint-Jean-Baptiste of Château-Gontier priory and and the castellian emancipation of the lord of this place. It characterizes two different periods in the eleventh century. The first half of the century is marked by the ascendancy of the count of Anjou, initial donor of the curtis of Bazouges to the monks and to his villicus, Gontier. Between 1001 and 1060, the count manoeuvres to preserve his seizure: he manipulates the opposition of the monks and the castellian... and imposes his mediation between them. During the second half of the century, the death of Geoffoy Martel marks the weakening of the countal authority and reinforces the opposition between the monks and the dominus of Château-Gontier. The religious places the quarrel on the gregorian ground and support their position on their friendship with the Mathefelon (the local rivals of the lord). The riposte of the lord consolidates his position: to counterbalance the religious of Saint-Aubin, he establishes, in Azé, a rival priory, which depends of Saint-Nicolas. The priories played an essential part for the emancipation of the dominus of Château-Gontier. 
